\title{
Application of Image Processing on Segmentation of the EUV
}

\section{Solar Images}

\author{
Hemamalini G $\mathbb{E}^{1}$, Dr. Kariyappa $\mathbb{R}^{1}$, Dr. J Prakash ${ }^{2}$
}

1, Vemana Institute of Technology,Koramangala,Bangalore-34,9886645402, 9449786885

2, Bangalore Institute of Technology, V.VPura,Bangalore-1,9845070991

\section{Abstract}

Recent trends in image processing are more focused on new technologies involved in the field of Astronomy, Astrophysics and Medical field applications. Image processing in astronomy applies different algorithms to extract scientific results from the observed data. Through image processing, coronal features of Sun are extracted and studied. The extraction of different coronal features and determination of their contributions to EUV irradiance variability are important in heliophysics, the Earth's climate and space weather applications. The objective of this research is to extract the coronal Active Regions (ARs), Coronal Holes (CHs) and Quiet Sun (QS) from the full - disk calibrated daily images of $174 \mathrm{~A}^{\circ}$ observed from PROBA2/SWAP instrument for period of 6 months (October 2016 - March 2017). In image processing the enhancement and different segmentation techniques have been applied. Based on these feature extraction the Support Vector Machine (SVM) classification is implemented. The cumulative intensity values of all the features are derived. The contribution of these features to full-disk integrated EUV irradiance variability are estimated and found that the active regions contribute up to $60 \%$ whereas the quiet sun and coronal holes will be around 38\% and 2\% respectively. In addition, these results suggest that the variations in intensity of the various coronal features have to be taken into account in irradiance models. Furthermore the SVM classification method has been estimated and the result presents a 90 percent accuracy.

Key words: Active Regions (ARs), Coronal Holes (CHs), Quiet Sun (QS), Histogram Equalization, Canny detection and SVM. 


\section{Introduction}

Visual information and images play an important part in our daily lives. Image processing is used in different applications like medical imaging modalities i.e. X-ray/ ultrasound, security, remote sensing and astronomy \& astrophysics. In astronomy, in particular, the study of the Sun-Earth interaction is extremely important in our daily technology dependent society. The image processing techniques that are used in automated feature detection algorithms applied to solar data are image enhancement, automated detection of spatial features, imagery and forecasting.

The Sun is the main source of energy and is governed by the magnetic field structure, emits radiation and plasma. To improve forecasting models of space weather and to develop better early warning system, there should be understanding of solar activity processes such as solar flares, sunspots, filaments and coronal mass ejection. The automatic image processing enables detecting and tracking various features in the solar atmosphere, this is essential to support studies of solar activity, solar evolution, climate change and understanding space weather. To study the spatial and temporal variations of the features a large number of solar images for a longer period are required. The spatial features are time dependent and their dynamics can be investigated based on high resolution time series of observations of the Sun. The different layers of the Sun enable to analyze and measure the different features of the Sun. The different layers of the Sun are shown in the Figure 1.1[1] [2].

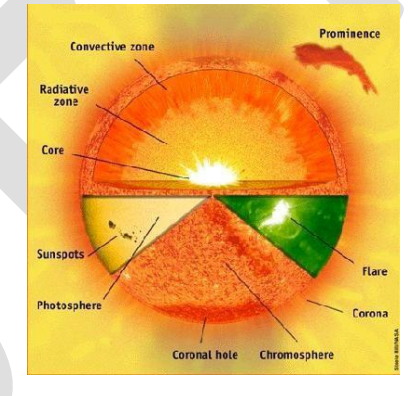

Figure 1.1: Different Layers of Sun

The Sun is divided into two parts, namely the solar interior and atmosphere. The solar interior consists of (i) the inner core; (ii) the radiative zone, (iii) the convection zone. Whereas the solar atmosphere consists of (i) the photosphere - visible layer, (ii) the chromosphere and (iii) the corona - outer most layer of the Sun. The solar atmosphere is highly structured with strong magnetic field and it is an inhomogeneous in nature. The photosphere is a thin visible layer of the Sun having temperature of about $6000 \mathrm{deg}$ C. The important feature observed in the photosphere are sunspot, granules and dark filaments[3]. The 
chromosphere is an irregular layer above the photosphere where the temperature rises from $6000^{\circ} \mathrm{C}$ to about $20,000^{\circ} \mathrm{C}$. The chromosphere is more visually transparent than the photosphere. The most common solar feature within the chromospheres are spicules, bright plage, super granules, network and bright points. The corona is the Sun's outer atmosphere. The corona displays a variety of features including active regions, coronal holes, coronal bright points, streamers, plumes and loops. These features change as a function of time and from eclipse to eclipse and the overall shape of the corona changes with the sunspot cycle.

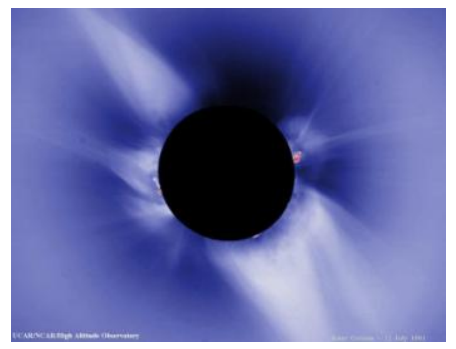

Figure 1.2: Solar Corona

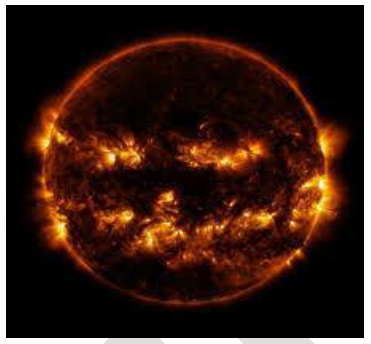

Figure1.3: Active Regions

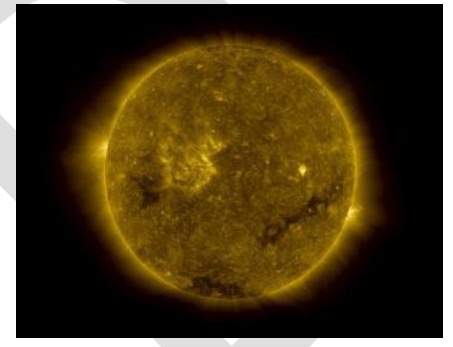

Figure 1.4: Quiet Sun

The active regions are brighter with higher intensity and magnetic field regions of the corona. The coronal holes are the areas where the Sun's corona is colder, hence darker, and has lower-density plasma than average because there are lower energy and gas levels. Coronal holes are part of the Sun's corona and are constantly changing and reshaping because the corona is not uniform. The coronal regions which are not a part of active regions and coronal holes are commonly identified as the quiet sun [4][5].

It is an important and difficult task to segment precisely the different coronal features because of their complex in nature. The segmentation of these coronal features is an important and useful process to understand the solar EUV \& UV irradiance variability. The coronal features such as the active regions, coronal holes and the quiet sun regions will contribute significantly to EUV \& UV irradiance variations and hence the feature extraction and segmentation in image processing will play important role to understand the various features and the processes happening in the Sun[6][7]. The different research methodologies and image processing techniques have been applied to coronal images to segment and extract the active regions, coronal holes and quiet Sun (Kumara, Kariyappa, Zender et al. 2014; Zender, Kariyappa, Giono, et al. 2017)[10][11]. The method implemented was SPoCA (Spatial Possibilistic Clustering Algorithm) is a set of multichannel fuzzy algorithm that automatically segment solar EUV images into a set of features. The SPoCA algorithm perfectly separates active region, quiet sun, and coronal hole classes through the minimization of a fuzzy intra-class variance[12]. 
The main objectives of this research are to use the spatially resolved images of the Sun observed from SWAP, EUV imager on PROBA2 space mission for duration of 6-months (Oct 2016 to March 2017) and different enhancement techniques have been applied on the SWAP images using MATLAB. Features of Sun are being extracted and segmentation is obtained using different edge detection methods. Contribution of features to EUV/UV irradiance variability is to be determined and discussed[8][9]. Measurement of full disk intensity of active regions, coronal holes and quiet sun for duration of 6 months is measured. The SVM (Support Vector Machine) technique is implemented to estimate the accuracy of the extracted features. This paper is organized as follows: Section 2 provides the proposed system design, Section 3 presents the different enhancement techniques and segmentation of edge detection algorithm and SVM classification, Section 4 discusses the results and discussion and Section 5 provides conclusions.

\section{Methodology, Techniques and their Implementation}
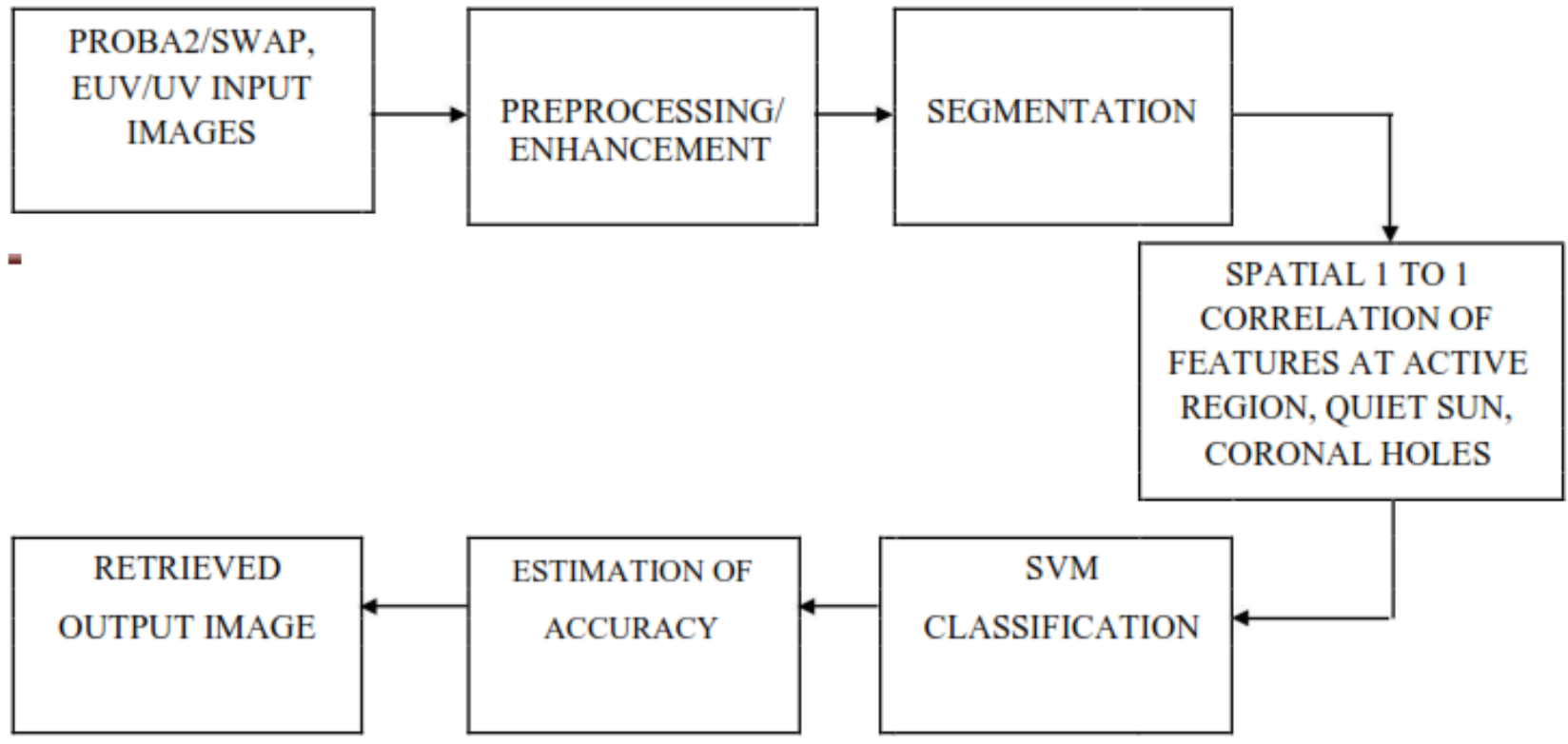

Figure 2: Block diagram

The 2-dimensional EUV \& UV images in 171A are observed from PROBA2/SWAP (Project for on board Autonomy/Sun Watcher Using Active Pixel System) space mission and its instrument. These images will be pre-processed, and enhanced using different image processing techniques. Various edge detection methods are used to identify the different solar coronal features like active regions (ARs), coronal holes 
$(\mathrm{CHs})$ and quiet sun regions (QS). These features will be identified and extracted from all the images based on their intensity values, mean and standard deviation. Canny edge detection which defines edges as zero crossings of second derivatives in the direction of the greatest first derivative is implemented. Segmentation partitions the image into distinct regions based on intensity, and similar attributes are considered. SVM classification is performed, which classifies the different features and based on this classification the accuracy of the extracted features is obtained. Segmented coronal features derived from the images corresponding to different heights in the solar atmosphere will be related.

The images commonly involves removing low-frequency background noise, normalizing the intensity of the individual pixel values, removing reflections, and masking portions of images. The several preprocessing techniques namely i) Contrast stretching ii) Log transformation iii) Power law transformation iv) Histogram equalization have been applied to solar images as shown in Figures 3.1 \& 3.2.

\section{Contrast stretching.}

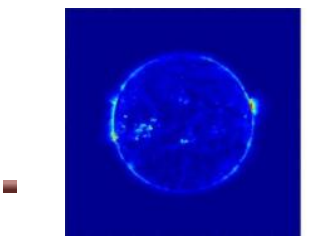

Figure 3.1: Original Image

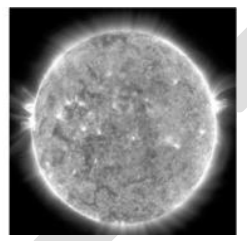

Contrast Stretching

Power transformations.

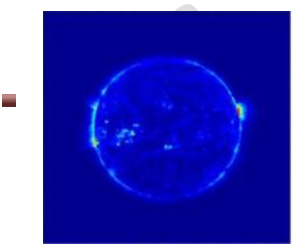

Figure 3.2: Original Image Power Law Transformation

\section{Log transformations}

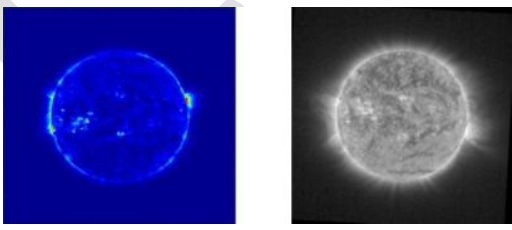

Original Image Log Transformation

Histogram equalization

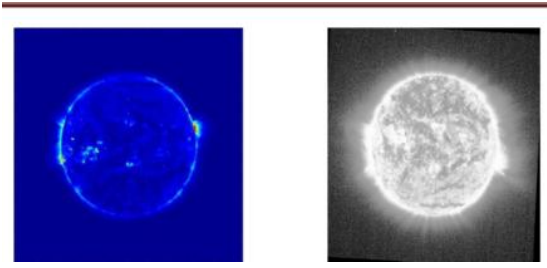

Original Image Histogram Equalization

The edge detection techniques have been applied and includes a variety of mathematical methods that aim at identifying points in a digital image at which the image brightness changes sharply or, more formally, has discontinuities. The different edge detection techniques have been implements such as Canny, Sobel, Robert and Perwits detection as presented in Figures 3.3 and 3.4. 


\section{Canny edge}

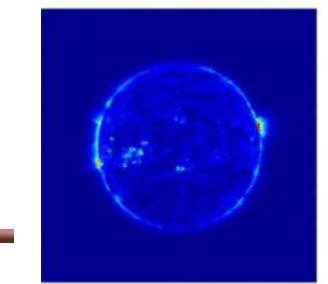

Figure.3.3: Original Image

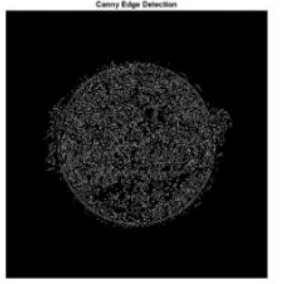

Canny Edge Detection
Robert edge detection

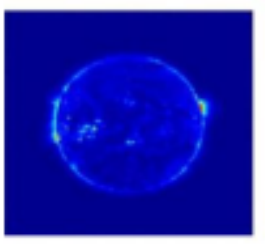

Figure .3.4 Original Image Roberts Edge Detection

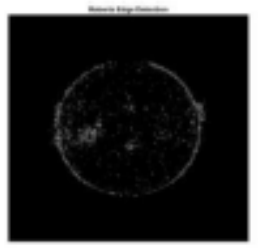

\section{Sobel edge}

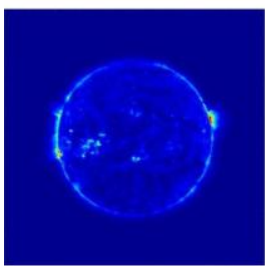

Original Image

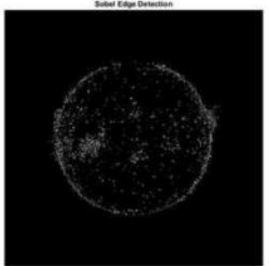

Sobel Edge Detection
Prewitt Edge

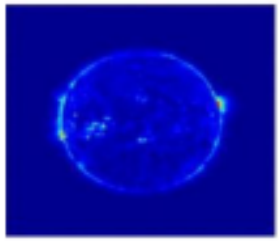

Original Image

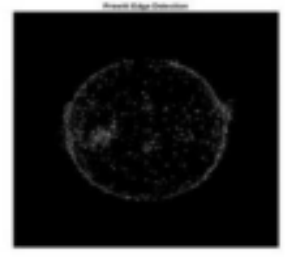

Prewitt Edge Detection

Extracted region of interests (ROI) such as ARs, CHs and QS are shown in the Figure 3.5.

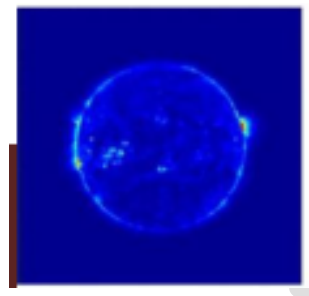

Figure. 3.5 Original Image

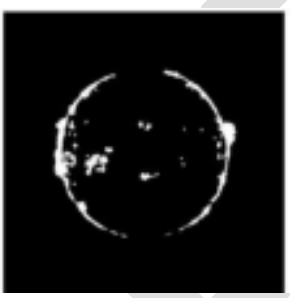

Extracted Active Regions

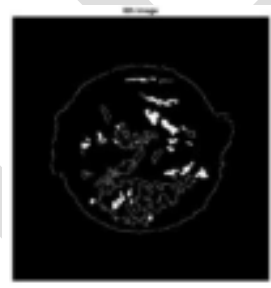

Extracted Coronal Holes

The Support Vector Machine is a supervised machine learning algorithm which can be used for classification. It uses a technique called the kernel trick to transform the data and then based on these transformations it finds an optimal boundary between the possible outputs. The main idea behind SVM is to find an optimal separating hyperplane with maximized margin. The maximum margin reduces the empirical risks (training errors) and causes a very good generalization performance. SVM has ability in generalization and good performance in pattern recognition (digit recognition, computer vision, and text \& speech categorization, etc.)[13].Linear Classifiers Support vector machines are an example of a linear twoclass classifier. The data for a two-class learning problem consist of objects labeled with one of two labels corresponding to the two classes; for convenience the labels are +1 (positive examples) or 1 (negative examples). In what follows, boldface x denotes a vector with components xi. The notation xi will denote the ith vector in a dataset composed of $\mathrm{n}$ labeled examples (xi,yi) where yi is the label associated with xi. 
The objects xi are called patterns or inputs, the inputs belong to some set X. A key concept required for defining a linear classifier is the dot product between two vectors, also referred to as an inner product or scalar product, defined as wT x $1 / 4 \mathrm{P}$ i wixi

A linear classifier is based on a linear discriminant function of the form

$$
\mathrm{f}=\mathrm{P} 1 / 4 \mathrm{X} \text { w } \mathrm{x} \text { p b: } 1 / 2
$$

The vector $\mathrm{w}$ is known as the weight vector, and $\mathrm{b}$ is called the bias. Consider the case $\mathrm{b}=0$ first. The set of points $\mathrm{x}$ such that wT $\mathrm{x} 1 / 40$ are all points that are perpendicular to $\mathrm{w}$ and go through the origin $-\mathrm{a}$ line in two dimensions, a plane in three dimensions, and more generally, a hyperplane. The bias b translates the hyperplane away from the origin. The hyperplane divides the space into two according to the sign of the discriminant function $\mathrm{f}(\mathrm{x})$ defined in Equation(1) [14]. The advantages of support vector machines are effective in high dimensional spaces, effective in cases where number of dimensions is greater than the number of samples, Uses a subset of training points in the decision function (called support vectors), so it is also memory efficient.

\section{Results and Discussion}

We have done an analysis on six-months (October 2016 - March 2017) daily full-disk 174 A solar images, which are obtained from PROBA2/SWAP instrument. The different coronal features such as ARs, CHs and QS have been segmented using different techniques based on their intensity threshold values. The cumulative intensity values of the features are extracted. The contribution of AR, CH and QS to full-disk intensity and time series of various features like active regions, coronal holes and quite sun are plotted for a period of 6 months and are presented in the Fig 3.1-3.3. The intensity time series of all the features show clearly the solar activity and in addition they show a 27-day solar rotation. In Figures 3.4 - 3.6 we have plotted the intensity of ARs, CHs and QS as a function of sunspot number and they show a fairly good correlation. We have estimated the contribution of ARs, CHs and QS to total integrated full-disk intensity of $174 \mathrm{~A}$ image and found that the ARs contributes upto 60\%, whereas the QS and CHs are contributing $38 \%$ and $2 \%$ respectively. The time series of full-disk intensity and variation in contribution of ARs, CHs and QS are shown in Figures 3.7 - 3.10. It is noticed that the contribution plots also show a 27-day rotational modulation. 


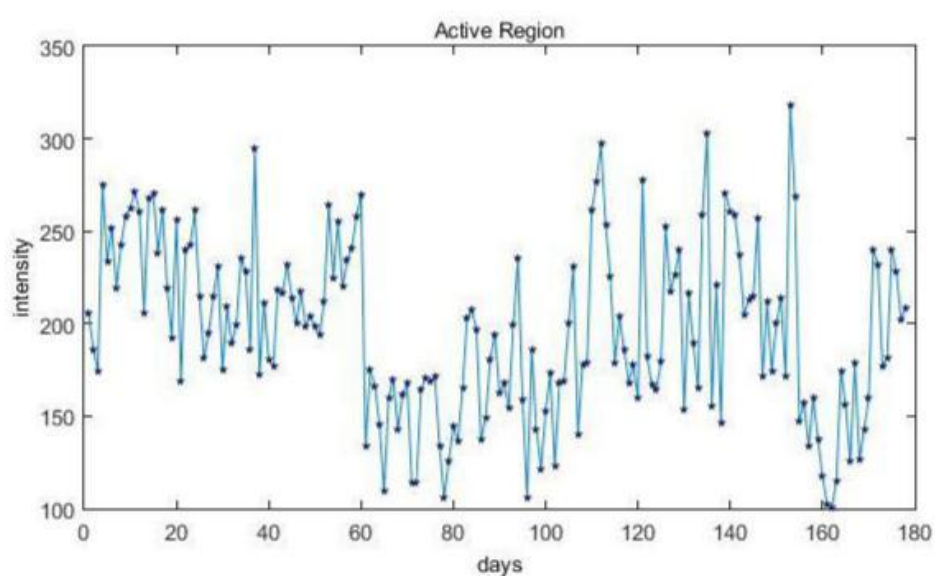

Figure 3.1: Intensity variation of active regions

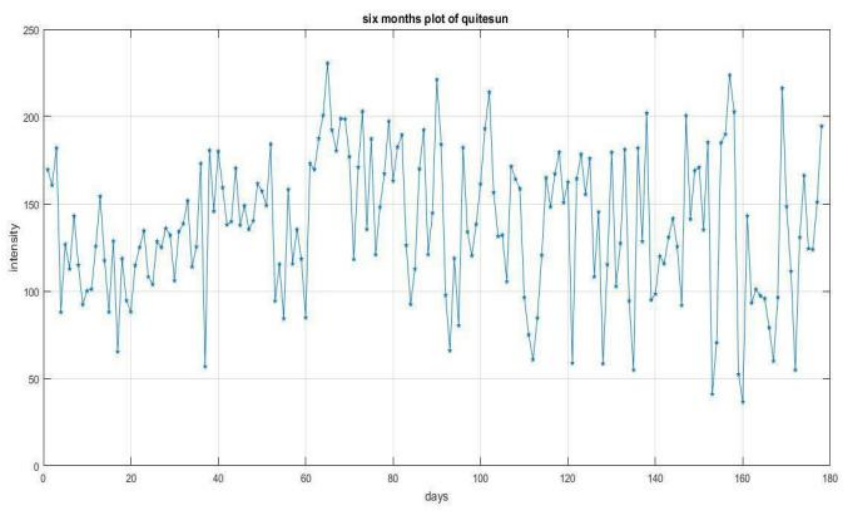

Figure 3.3: Intensity variation of quite sun

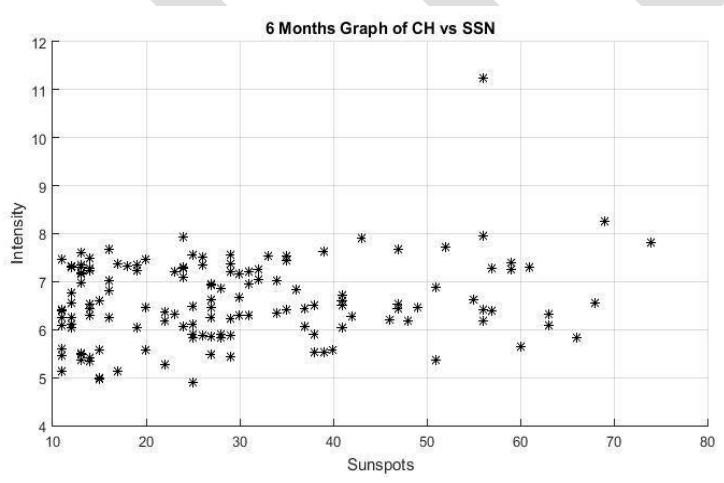

Figure 3.5: Scattered plots CH Vs SSN

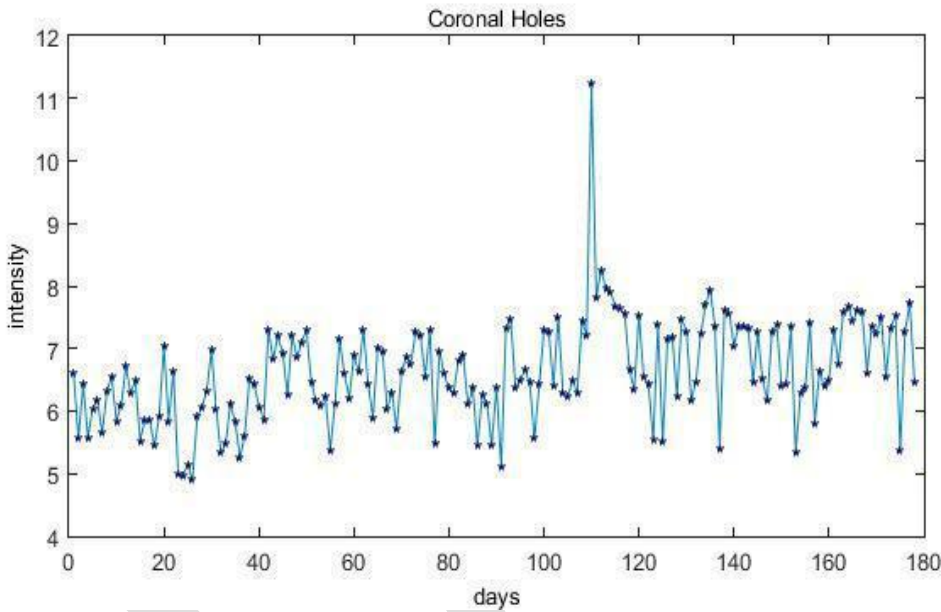

Figure 3.2: Intensity variation of coronal holes

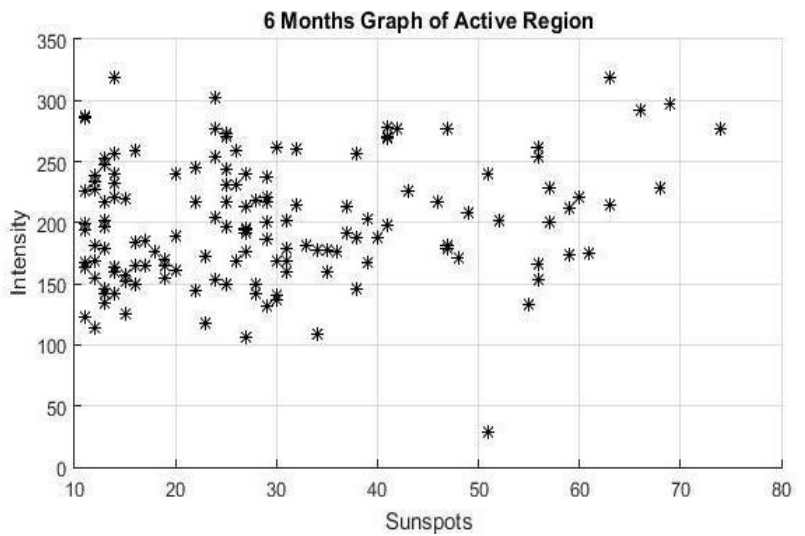

Figure 3.4: Scattered plots AR Vs SSN

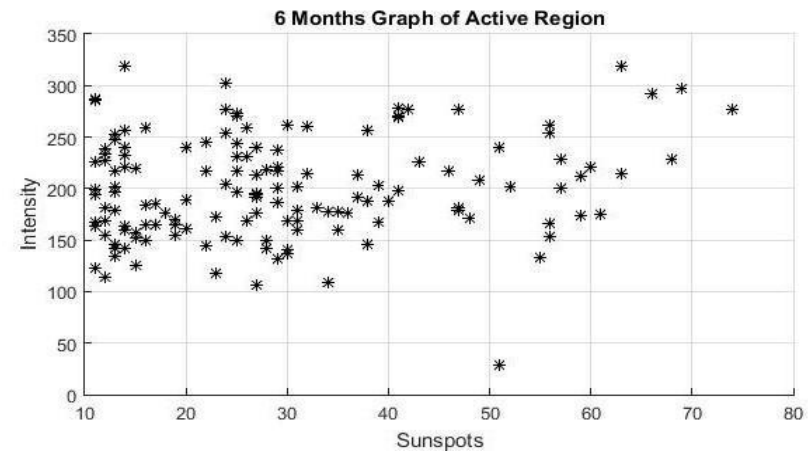

Figure 3.6: Scattered plots QS Vs SSN 


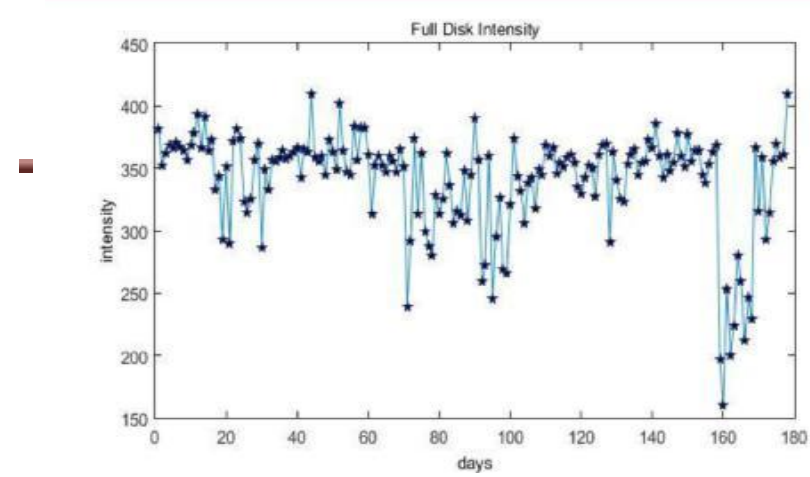

Figure:3.7: Variation of Full disk intensity

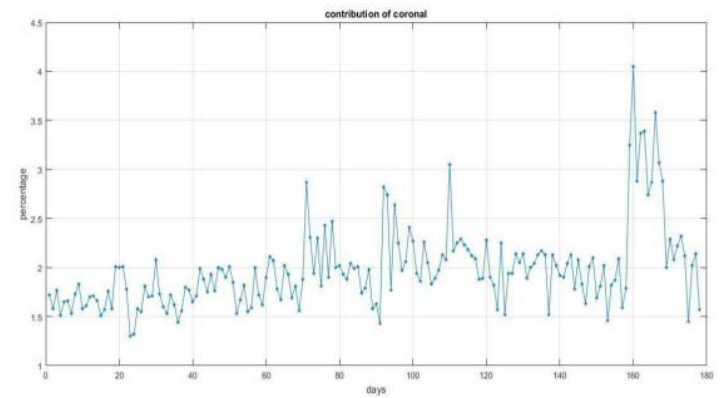

Figure 3.9: Variation of Contribution of coronal holes

\section{SVM RESULTS}

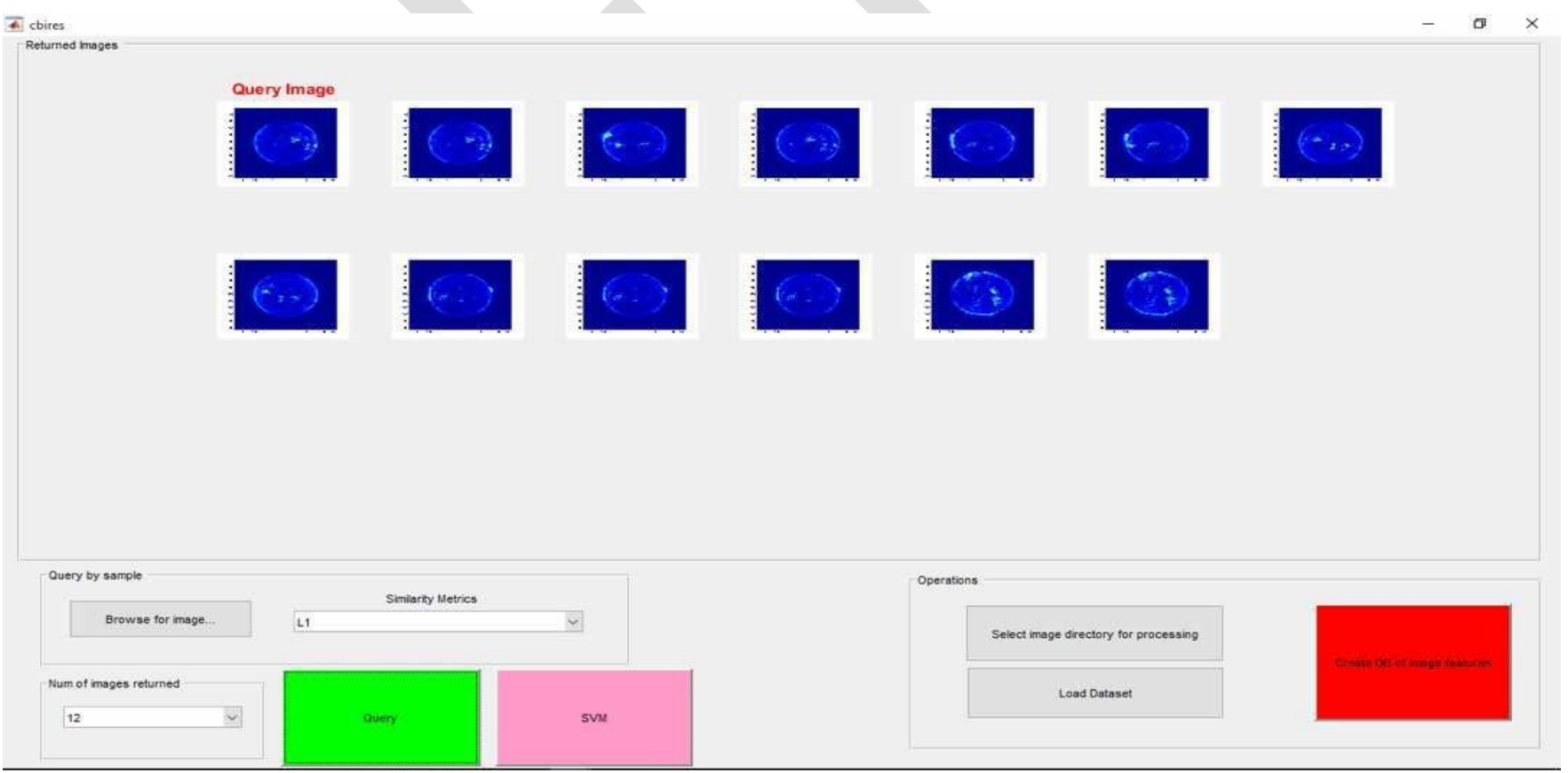

Figure 3.11: SVM technique

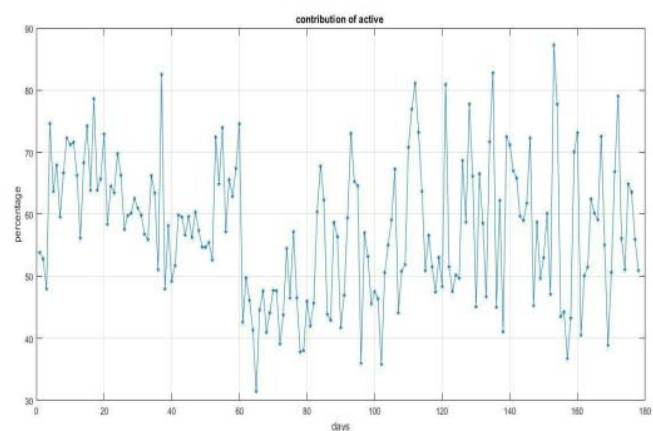

Figure 3.8: Variation of Contribution of active region

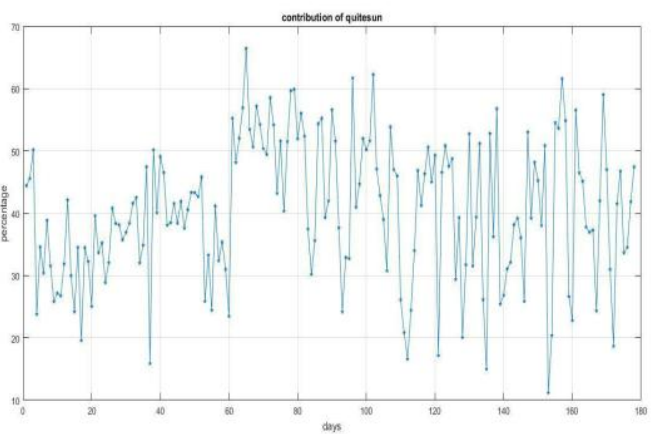

Figure 3.10: Variation of Contribution of quiet sun 


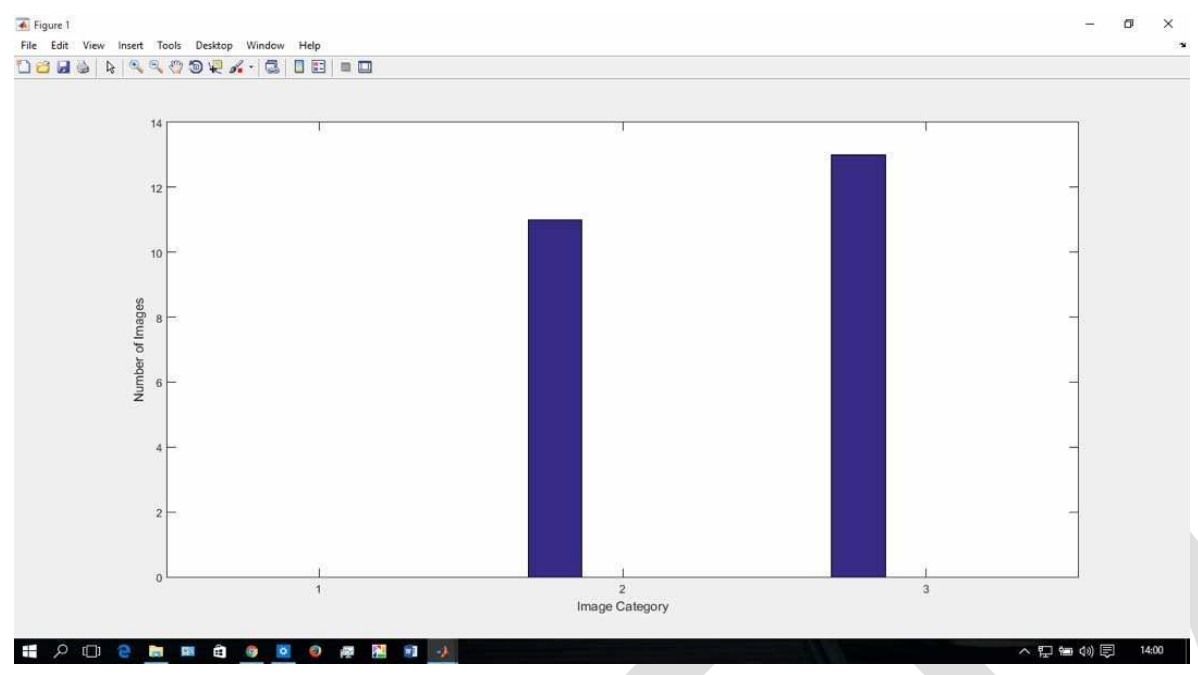

Figure 3.12: SVM results

\section{Conclusion}

In this research, analysis was performed on 174 A images obtained from SWAP, EUV imager on PROBA2 Space Mission for a period of 6 months (October 2016 - March 2017). Various coronal features like the Active regions, Coronal holes, and the Quiet Sun are identified, segmented and extracted using image processing techniques in MATLAB. Enhancement, segmentation using different techniques was implemented and the best technique such as Histogram Equalization and Canny detection algorithm was considered. ROI (Region of interest) methods were used to emphasize and highlight these regions. The cumulative intensity of the Active regions, Coronal holes and the Quiet Sun for a period of 6 months (October 2016-March 2017) are calculated and time series of the respective regions are generated. The contribution of the Active regions, Coronal holes and the Quiet Sun to the total UV flux was estimated. The active regions have contributed up to $60 \%$ whereas the quiet sun and coronal holes contributed around $38 \%$ and $2 \%$ respectively. The time series of various features like Active regions and Coronal holes are shown in table 3.1 to 3.3 for the total duration. The scatter plot for each of these comparisons is presented in Fig 3.4 to Fig 3.6. The graphical representation of the intensity of the three region with respect to time and the comparison of the three region with SSN ( Sun spot numbers) are given in graph Fig 3.7 to Fig 3.11. The result of the SVM technique is shown in Fig 3.12 which determines a 90 percent accuracy of classification obtained. 


\section{References}

[1]V. Barra, V. Delouille, M. Kretzschmar, and J. -F. Hochedez, "Fast and robust segmentation of solar EUV images: algorithm and results for solar cycle 23", A\&A, vol. 505, pp. 361-371, October 2009.

[2]D. Berghmans, J. -F. Hochedez, J. M. Defise, et al. "SWAP onboard PROBA 2, a new EUV imager for solar monitoring", Advances in Space Research, vol. 38, pp. 1807- 1811, Jan. 2006.

[3]R. Kariyappa, "CaII K Imaging to Understand UV Irradiance Variability", Journal of Astrophysics and Astronomy, vol. 21, pp. 293-297, June 2000.

[4]R. Kariyappa, "Spatially resolved images and solar irradiance variability", Journal of Astrophysics and Astronomy, vol. 29, pp. 159-162, March 2008.

[5]Kariyappa, R. \& Pap, J. M. 1996, Sol. Phys., 167, 115

[6]S.T. Kumara, R. Kariyappa, M. Dominique, et al., "Preliminary Results on Irradiance Measurements from Lyra and Swap", Advances in Astronomy, vol. 2012, pp. 5-10, 2012.

[7]S.T. Kumara, R. Kariyappa, J.J. Zender, et al., "Segmentation of coronal features to understand the solar EUV and UV irradiance variability", A\&A, vol. 561, pp. A9-A18, Jan. 2014.

[8]A. A. Pevtsov, G. H. Fisher, L. W. Acton, et al., "The Relationship Between X-Ray Radiance and Magnetic Flux", ApJ, vol. 598, pp. 1387-1391, Dec. 2003.

[9]J.R. Worden, O.R. White, and T. N. Woods, "Evolution of Chromospheric Structures Derived from Ca II K Spectroheliograms: Implications for Solar Ultraviolet Irradiance Variability" ApJ, vol. 496, pp. 9981014, March 1998.

[10]M.S. Yalim, and S. Poedts, "Variations in EUV Irradiance: Comparison between LYRA, ESP, and SWAP Integrated Flux", Advances in Astronomy, vol. 2014, pp. 957461, 2014.

[12]J.J. Zender, R. Kariyappa, G. Giono, et al., "Segmentation of photospheric magnetic elements corresponding to coronal features to understand the EUV and UV irradiance variability", A\&A vol. 605, pp. A41-A52, Sept. 2017.

[13]Jing Zhang, Xue-dong Zhang, Seok-wun Ha, "A Novel Approach Using PCA and SVM for Face Detection", Fourth International Conference on Natural Computation 2008 IEEE

[14] W. Xiaodan and W. Chongming, Using membership function to improve multi-class SVM classification, ICSP Proceeding, China, 2004. 
DOI : https://dx.doi.org/10.26808/rs.ed.i7v6.12

\section{Acknowledgement}

The authors would like to thank the PROBA2 science centre, located at the Royal Observatory of Belgium (ROB) in Brussels for providing SWAP data. Hemamalini and Kariyappa would like to express their sincere thanks to Dr. B.G.Vijayasimha Reddy, Principal, Dr. T. Yella Reddy, Dean and rof.S.M.Chandrasekar, HOD, ECE for their encouragement and support on this research. The authors would be thankful to the project students (Manish, Ashwini,Divya and Jyothi) their help in the image analysis. 